\title{
Discrepancias entre el diagnóstico Clínico y Anatomo-Patoló- gico en el Hospital Escuela Universitario De Honduras.
}

'Bachiller en ciencias y letras. Estudiante de Medicina, $V$ año, Facultad de Ciencias Médicas; Universidad Nacional Autóno-

2 especialista en Epidemiología; Departamento de Salud Pública, Facultad de Ciencias Médicas. Docente de la cátedra "Salud pública III" en la Facultad de Ciencias Médicas, Universidad Nacional Autónoma de Honduras.

2 Médico, especialista en Patología, Departamento de Patología, Hospital Escuela Universitario. Coordinador y docente de la cátedra de "Patología II" en la Facultad de Ciencias Médicas, Universidad Nacional Autónoma de Honduras.

Correspondencia a: Ana Margarita García Reyes Correo electrónico: ana.mar.9@ hotmail.com

Palabras clave: autopsia, clasificación de Goldman, discrepancias clínico-patológicas

Keywords: autopsy, Goldman's classification, clinico-pathologic discrepancies.

\section{Discrepancies between the Clinical and Anatomy-Pathological diagno- sis at the Hospital Escuela Universitario in Honduras.}

Ana Margarita García Reyes ${ }^{1}$, Ana Gabriela Alberty Murillo ${ }^{1}$, Angie Nicole Avila Matute Daniela Michelle Seaman Peñalva', Iván Alexy Espinoza Aguiriano ${ }^{1}$, Jose Carlos Zavala Carranza ${ }^{1}$, Maria Jose Mezquita Ham ', Monica Maria Valenzuela Mairena', Pedro Antonio Hernandez Cruz', Sharon Betsabé Avilés Pinto', Walter Jauregui Alvarado', Dra. Gloria del Carmen Rivera Medina², Dr. César Augusto Durán López ${ }^{3}$

\section{RESUMEN}

La calidad diagnóstica es el resultado de integrar el conocimiento médico y reconocimiento de los errores clínicos, se alcanza únicamente con la identificación de las causas de muerte; es la correlación clínico patológica la herramienta principal para dicha acción. El objetivo general de la investigación fue determinar la discrepancia clínico-patológica y su relación con otras variables en las autopsias realizadas en la institución. Se revisaron I59 protocolos de autopsia del período comprendido entre enero 2012 y junio 2016, elaborados por el Servicio de Patología del Hospital Escuela Universitario de Tegucigalpa, Honduras. Se excluyeron 36 por no cumplir los criterios de inclusión. Se utilizaron la CIE-I0 y la clasificación de Goldman et al. para clasificar las patologías y establecer las discrepancias diagnósticas, respectivamente. El sexo predominante fue el femenino (2,96:I), la edad media fue de 38 años; prevalecieron los diagnósticos de embarazo/parto/puerperio y enfermedades infecciosas y parasitarias. Concluimos que en $46 \%$ de los casos existe discrepancia diagnóstica y la glomerulonefritis fue la principal causa de error, seguida de bronconeumonía. Se recomienda estandarizar el protocolo de autopsias y promover sesiones clínico-patológicas periódicas e integrales.

\section{ABSTRACT}

Diagnostic quality is the result of the integration of medical knowledge and recognition of clinical error, achieved only by identifying the cause of death; clinical pathological correlation is the primary tool for this action. The overall objective of this research was to determine clinical pathological discrepancy and its relationship with other variables within the autopsies performed at the institution. 159 autopsy protocols, elaborated by the Department of Pathology of Hospital Escuela Universitario in Tegucigalpa, Honduras, from January 2012 to June 2016, were reviewed. 36 were excluded for not meeting the inclusion criteria. ICD-I0 and Goldman et al. modified by Battle criteria were used to classify diseases and establish diagnostic discrepancies, respectively. The majority of patients were female (2.96:I), the mean age was 38 years old; diagnoses of pregnancy/birth/puerperium and infectious and parasitic diseases prevailed.We conclude that diagnostic discrepancies exist in $46 \%$ of all cases and glomerulonephritis was the leading cause of error, followed by bronchopneumonia. It is recommended that autopsy protocols be standardized, and integrative clinical pathological sessions are promoted and integral.

Procedencia y arbitraje: $\mathrm{no}$ comisionado, sometido a arbitraje externo.

Recibido para publicación: 04 de Agosto del 2016 Aceptado para publicación 02 de Dlciembre del 2016

Citar como: Rev Cient Cienc Med 2016;19(2): 20-26

\section{INTRODUCCIÓN}

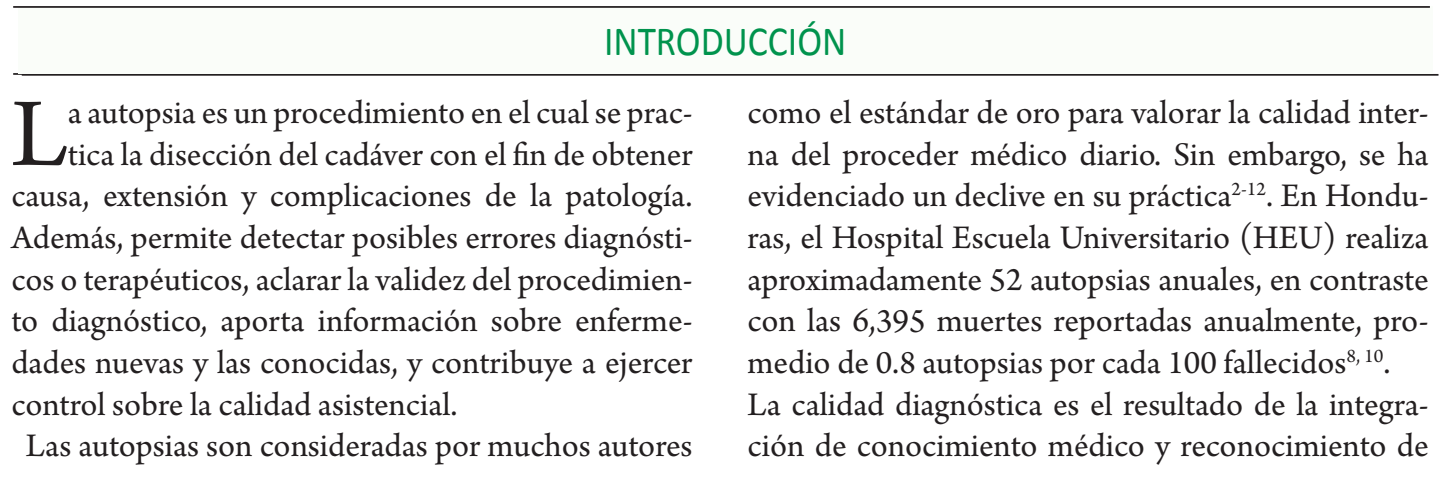


los errores clínicos; el cual se alcanza únicamente con la identificación de las causas de muerte; la correlación clínico patológica es la herramienta principal para dicha acción ${ }^{7}$.

El diagnóstico clínico es un proceso sistemático propenso a errores, estos pueden tener repercusiones en el manejo del paciente, si éstas son leves, solamente traerá como consecuencia un paciente insatisfecho que ocasionará la solicitud de cambio de médico, pero a medida que el error aumenta en complejidad podría llevar al paciente hasta su muerte ${ }^{12}$.

El propósito de la investigación es determinar la prevalencia de discrepancias entre el diagnóstico clínico final y la causa básica de muerte, utilizando la escala de GOLDMAN y col. modificado por Battle y casos no clasificables. La cual está dividida en discrepancias mayores y menores, cada una de estas dividida en clases; en las discrepancias mayores y 3 en las menores 2 . Estas clases son referentes al impacto en la evolución y pronóstico de la enfermedad si esta hubiera sido diagnosticada en otro momento o bajo otras circunstancias $^{11}$. Además, especificar las patologías que causan mayor mortalidad en adultos, las más frecuentemente mal diagnosticadas, y cuántas pudieron ser prevenibles con un mejor diagnóstico. Se determinará las salas con mayor prevalencia de errores diagnósticos y la utilidad de los métodos complementarios al momento de realizar un diagnóstico clínico certero. La investigación pretende crear un impulso para el personal de salud; que otorgue la importancia necesaria al diagnóstico integral y a las autopsias, como un proceso que debe ser frecuente y necesario para beneficio de la institución.

Así mismo, estimular a los estudiantes a exigirse al momento de realizar las historias clínicas, resaltar la seriedad de su componente legal y mostrar su indispensable uso para cumplir con la principal finalidad de todo médico; conservar la vida de los pacientes.

\section{MATERIALES Y MÉTODOS}

\section{Adquisición de la Información}

La investigación posee un alcance de tipo mixto; descriptivo y correlacional. Para la recolección de la información se utilizó protocolos proporcionados por el departamento de Patología del HEU. Se elaboró una ficha de recolección de datos, estructurada con 17 ítems diferentes.

Se extrajo información contenida en protocolos de autopsia de los últimos 5 años del Departamento de Patología del HEU para describir los datos generales de los pacientes; la consignación de los diagnósticos que se realizaron en el transcurso de la estancia intrahospitalaria del paciente: de ingreso y clínico final; los realizados en el estudio post-mortem: diagnóstico macroscópico y microscópico; causa básica, intermedia e inmediata de muerte. Así como, identificar las principales causas de muerte diagnosticadas según clínica y por autopsia.

La hipótesis planteada más relevante fue: La discrepancia entre el diagnóstico clínico y el post mortem es del 40-45\% del total de pacientes con autopsia en el Hospital Escuela Universitario.

Cálculo de la Muestra

Con una población total de 272 protocolos en el periodo Enero 2012-Junio 2016, se utilizó el programa de cálculo de tamaño de muestra STATS ${ }^{\circledast}$, para encontrar una muestra representativa, con un porcentaje de error máximo de $5 \%$ y un nivel de confianza de 95\%; el instrumento se aplicó en una muestra de 159 protocolos de autopsias en el departamento de patología; posteriormente se calcularon las muestras por año según el total de protocolos.

Criterios

Se consideró como criterios de inclusión pacientes mayores de 18 años, pacientes con diagnóstico clínico y diagnóstico patológico post mortem, y como criterio de exclusión a pacientes menores de 18 años, pacientes con reportes cuya información era imprecisa y protocolos en los cuales faltaron el diagnóstico clínico final o la causa básica de muerte.

Análisis Estadístico

Una vez recaudada la información, los datos fueron procesados por métodos computarizados mediante el paquete de programas estadísticos " $R$ ". La presentación de los resultados se efectuó en tablas confeccionadas en el programa de computación Excel. La significación estadística de 4 tablas se evaluó mediante la prueba exacta de Fisher. En general, las evaluaciones de significación se basaron en valores $\mathrm{P}$ a dos caras. Los valores de $\mathrm{P}, 0,05$ fueron considerados como los valores p significativos, 0,1 como límite significativo.

\section{RESULTADOS}

El total de defunciones entre enero del 2012 hasta junio del 2016 es de 13 074, se realizó autopsia a 319 pacientes $(2,44 \%)$. El universo fue de 272 protocolos, con una muestra de 159 protocolos; 36 protocolos fueron excluidos incumplimiento de criterios. El año donde más se realizó autopsias fue en el 2012 (3,39\%), el sexo predominante fue el femenino (74,6\%). La edad media fue de 38 años.

Diagnósticos clínicos y post mortem.

El 98\% de protocolos contenía diagnóstico de ingreso donde prevalecían los diagnósticos de embara- 
zo/parto/puerperio, enfermedades infecciosas y parasitarias y enfermedades genitourinarias. El motivo principal para que se realizara autopsia fue por petición de familiar (50\%) seguido de un $44 \%$ por causas reglamentarias.

Se consignó siempre el diagnostico macroscópico, en contraste con el diagnostico microscópico, siendo el año 2015 cuando más se realizó (39\%). Se verifica la realización de diagnóstico microscópico con el año $(p=0,000)$. Las principales causas de muerte según diagnóstico clínico final son síntomas/signos/ hallazgos anormales clínicos y de laboratorio no clasificados en otra parte, enfermedades del sistema respiratorio y enfermedades infecciosas y parasitarias. Mientras que embarazo/parto/puerperio, enfermedades del sistema respiratorio y tumores son las principales causas de muerte según causa básica de muerte en los protocolos de autopsia. El 29\% de los protocolos reportaron hallazgos incidentales.

El diagnóstico clínico final según sexo (Ver Tabla 1), en mujeres: Embarazo/parto y puerperio, enfermedades del sistema respiratorio, enfermedades del sistema circulatorio y enfermedades infecciosas y parasitarias. En los hombres: Enfermedades endocrinas y metabólicas, enfermedades del sistema respiratorio, y enfermedades infecciosas y parasitarias. El rango de edad más prevalente fue de 19-26 años.

Las principales causas básicas de muerte según sexo (Ver Tabla 1) son embarazo/parto/puerperio, tumores y enfermedades del sistema respiratorio en mujeres; para los hombres son enfermedades del sistema respiratorio, enfermedades infecciosas y parasitarias, enfermedades del sistema circulatorio y del sistema digestivo. En los rangos de edad (Ver Tabla 1), las mayores frecuencias de causa básica de muerte son: Enfermedades del embarazo/parto/puerperio, enfermedades del sistema respiratorio en las edades de 19-26 años, seguido de tumores a las edades de 35-42 años.

En mujeres los diagnósticos secundarios (Ver Tabla 2) fueron: Sistema circulatorio, enfermedades endocrinas/metabólicas y causas extremas de morbilidad y mortalidad. En hombres fueron causas extremas de morbilidad y mortalidad, enfermedades del sistema circulatorio, enfermedades infecciosas y parasitarias, enfermedades del sistema respiratorio y por síntomas, signos y hallazgos clínicos anormales prevalecieron como causa inmediata de muerte (Ver Tabla 1).

Discrepancia Diagnóstica

Existe discrepancia diagnóstica del 46\%. Según la Clasificación de Goldman, la Clase V con mayor porcentaje, seguido de Clase II y Clase I (Ver Tabla 4). El año con mayor discrepancia diagnostica es el 2014
(66.7\%). Se verificó la correlación año con discrepancia diagnóstica $(p=0,025)$. La discrepancia entre el diagnóstico de ingreso y el diagnóstico clínico final es de $25,6 \%$. Se verificó la correlación año con discrepancia diagnóstica $(\mathrm{p}=0,025)$.

Los porcentajes de discrepancia en los servicios hospitalarios son Medicina Interna $(71,2 \%)$, Gineco-obstetricia (19,2\%), Cirugía (5,8\%), Neurología y Neurocirugía con un 19\% cada uno. El mayor porcentaje de defunción está en Medicina Interna en el año $2012(70,6 \%)$ al contrario del servicio de Gineco-obstetricia $(39,3 \%)$ en el año 2013. No se observó una correlación estadísticamente significativa $(p>0,05)$ entre las variables "servicio hospitalario" con "discrepancia diagnóstica” ( $p=0,849)$.

En todos los protocolos se consignó el uso de estudios complementarios básicos, estos presentan discrepancia diagnostica de $46.3 \%$. El uso de estudios de imagen aumentó un 17,2\% desde 2012 al 2016, utilizándose en un 90,9\% en el año 2016, la discrepancia en protocolos donde se usó métodos de estudio de imagen es de $78,9 \%$, los que no presentan discrepancia diagnóstica y estudio de imagen está representado por $78,8 \%$.

El diagnóstico macroscópico se consignó en todos los protocolos. En los protocolos de autopsia en los cuales sí se realizaron diagnóstico microscópico, la discrepancia diagnóstica está representada por $19,3 \%$, Se observó una correlación estadísticamente significativa $(\mathrm{p}<0,05)$ entre las variables "diagnóstico microscópico" con "discrepancia diagnóstica" $(\mathrm{p}=0,002)$.

Las patologías que con mayor frecuencia son mal diagnosticadas son la glomerulonefritis $(5,4 \%)$, la bronconeumonía $(5,4 \%)$, neumonía $(3,6 \%)$ y la meningitis aguda $(3,6 \%)$..

\section{DISCUSIÓN}

La autopsia cumple diversas funciones: Control sobre la calidad asistencial, docencia e investigación.13 En HEU el porcentaje de autopsias realizadas en relación a las muertes ocurridas por año es $2,44 \%$, se realizan poco más de dos necropsias por cada 100 personas fallecidas, un porcentaje muy pobre en comparación con el promedio de necropsias realizadas a nivel mundial, que oscila entre 22 y $38 \%{ }^{14}$. En los últimos años se ha reportado que la práctica de la autopsia ha declinado en un aproximado de $60 \%$ en países como Australia, Estados Unidos, Reino Unido, India; Francia, Canadá, China y Suiza 2-12, factores identificados en relación a su disminución a nivel mundial 


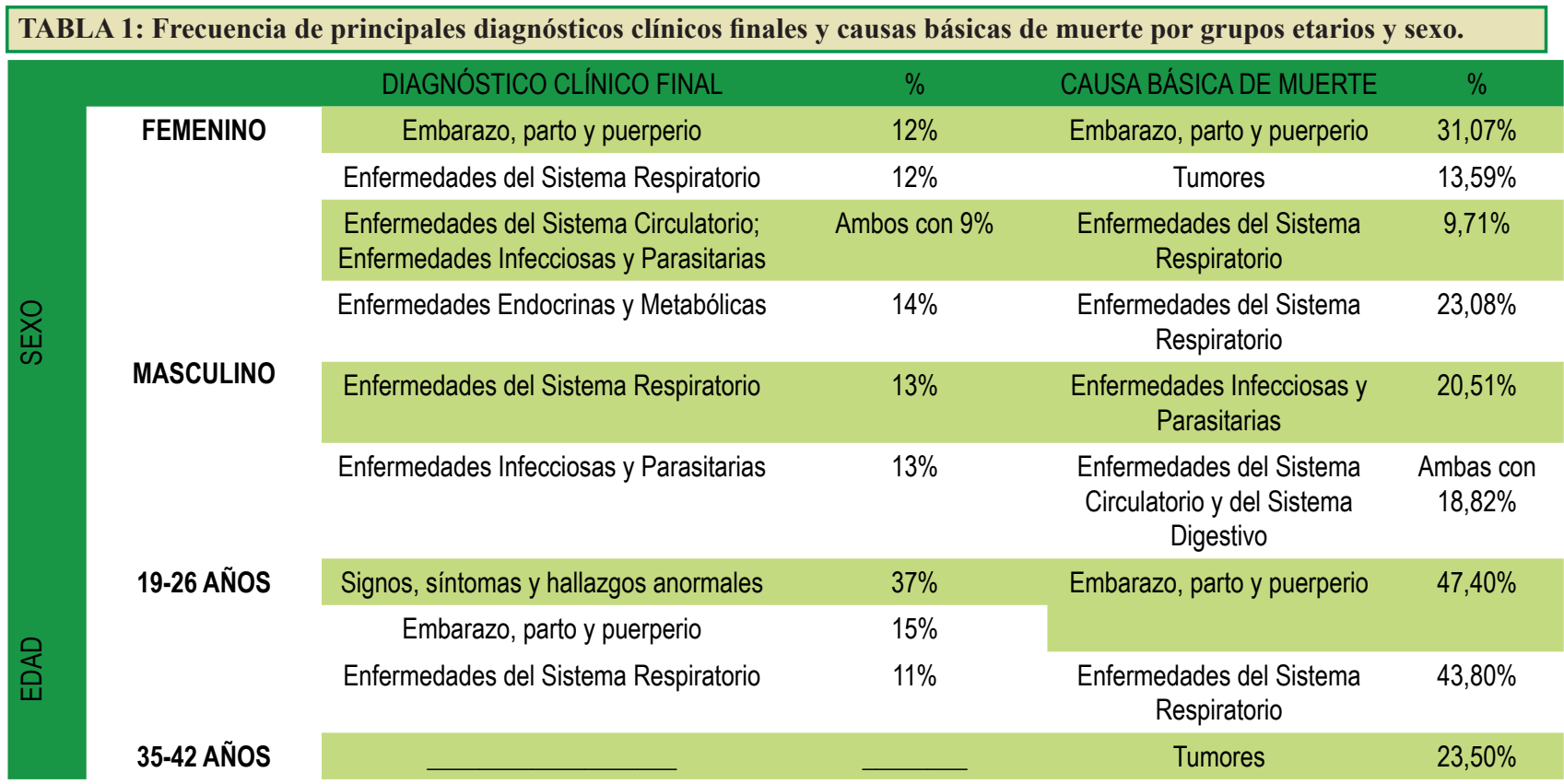

TABLA 2: Principales diagnósticos secundarios por sexo

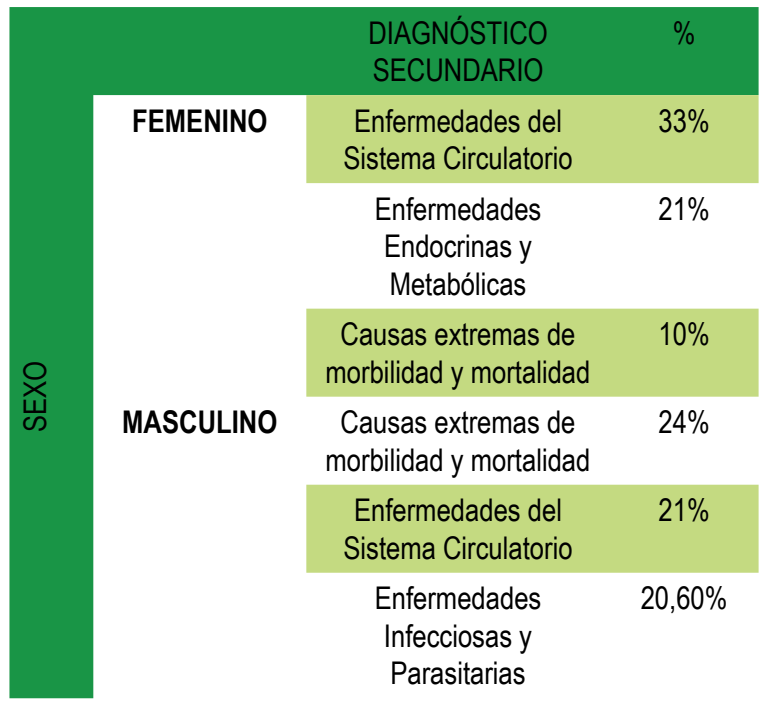

son: El uso y sobre confianza agregada a los de métodos de imágenes clínicos, costo institucional de la autopsia, falta de educación sobre los beneficios que representa la autopsia para las instituciones médico-estudiantiles, y la percepción de ser un procedimiento que impide el descanso del difunto ${ }^{8,14,15}$.

Estudios mundiales de discrepancia diagnóstica indican la prevalencia de necropsias en el sexo masculino y edades medias de 50-60 años, en Latinoamérica estas cifras varían, son poblaciones con ambos extremos de edad entre los 32-97 años ${ }^{2-10,15,16}$.En nuestro estudio el sexo femenino predominó sobre el masculino $(2,96: 1)$,la edad media es de 38 años, con un rango de 18-81 años.
Tabla 3: Frecuencia de principales causas inmediatas de muerte.

\begin{tabular}{cc}
\hline $\begin{array}{c}\text { CAUSA INMEDIATA DE } \\
\text { MUERTE }\end{array}$ & $\%$ \\
\hline $\begin{array}{c}\text { Enfermedad infecciosas y } \\
\text { parasitarias }\end{array}$ & $20,60 \%$ \\
\hline $\begin{array}{c}\text { Enfermedades del sistema } \\
\text { respiratorio }\end{array}$ & $19,40 \%$ \\
Síntomas, signos y \\
hallazgos clínicos \\
anormales
\end{tabular}

Tabla 4: Discrepancias diagnósticas de acuerdo a la Clasificación de Goldman.La discrepancia diagnóstica detectada en nuestro estudio fue de $43,9 \%$ de discrepancias mayores (Clase I: $21,1 \%$, Clase II: $22,8 \%$ ) y $10,6 \%$ de discrepancias menores. La concordancia fue valorada en la clase $V$ (sin discrepancia diagnóstica) siendo de $54,5 \%$.

$\begin{array}{ccc}\begin{array}{c}\text { CLASIFICACIÓN DE } \\ \text { GOLDMAN }\end{array} & \text { FRECUENCIA } & \text { PORCENTAJE } \\ \text { CLASE I } & 26 & 21,1 \% \\ \text { CLASE II } & 28 & 22,8 \% \\ \text { CLASE III } & 2 & 1,6 \% \\ \text { CLASE V } & 67 & 54,5 \% \\ \text { TOTAL } & 123 & 100 \%\end{array}$

Diagnósticos Clínicos y Post-Mortem

El diagnóstico de ingreso establece el tratamiento inicial del paciente, debe ser resultado de la evaluación clínica rigurosa, su relación con el diagnóstico clínico final es clave para valorar la calidad de los servicios receptores de pacientes, en la li- 


Abreviaturas utilizadas en este
artículo:
CIE-10 : Clasificación Interna-
cional de enfermedades décima
versión
HEU : Hospital Escuela Univer-
sitario
DCF : diagnostico clínico final
DSC: diagnostico secundario
CIM : causa inmediata de muer-
te
CBM: causa básica de muerte
EPOC: enfermedad pulmonar
obstructiva crónica

teratura su discrepancia varía de $25 \%-41 \%{ }^{4}$. En los protocolos revisados se consignó el diagnóstico de ingreso en casi todos (98\%), de este las enfermedades del embarazo/parto/puerperio (19,1\%), refleja el sexo más estudiado; las enfermedades infecciosas $(12,5 \%)$ y enfermedades genitourinarias $(9,2 \%)$ siguen en orden de frecuencia. La discrepancia entre el diagnóstico de ingreso y el diagnóstico clínico final fue de $25,6 \%$. Se verificó la correlación año con discrepancia diagnostica . Las enfermedades se clasificaron por sexo y grupos de edad dentro del diagnóstico clínico final (DCF), diagnóstico secundario (DSc), dentro de los diagnósticos clínicos; y causa inmediata de muerte (CIM) y causa básica de muerte (CBM) dentro de los diagnósticos postmortem (Ver Tabla 1, $2,3)$. Según la OMS, las principales causas de defunción en el mundo entre 2000-2012 fueron cardiopatía isquémica, los accidentes cerebrovasculares, infecciones de vías respiratorias inferiores y EPOC; estas concuerdan con estudios en Sri Lanka y España ${ }^{17,23,24}$. En estudios latinoamericanos en Colombia, Cuba y Costa Rica se encontró que las causas citadas de muerte fueron por shock, bronconeumonía, y enfermedades infecciosas, el infarto agudo de miocardio y las enfermedades neoplásicas ${ }^{15,16,18,25}$. Los principales diagnósticos clínicos finales en nuestro estudio están dentro de la categoría "signos, síntomas y hallazgos anormales clínicos y de laboratorio no clasificados en otra parte" (16,2\%), (Falla multiorgánica, choque hipovolémico, choque cardiogénico y hemorragias de vías respiratorias), enfermedades del sistema respiratorio y enfermedades infecciosas y parasitarias.

Los estudios sobre discrepancia concuerdan en que la realización de las autopsias se ha limitado únicamente con la presencia de dudas diagnósticas, enfocadas en las poblaciones pediátricas al considerarse inútil en la adulta y la concepción negativa de los familiares ${ }^{8,13,15}$. A pesar de estos datos, el 50\% de los familiares de los pacientes fallecidos, solicitaron autopsia. El 44\% se solicitó por autoridades del hospital o los mismos médicos, incluyéndose como causa reglamentaria.

Hubo similitud de causa básica de muerte entre nuestros hallazgos y los demás estudios, en relación a las enfermedades respiratorias, excepto en enfermedades de embarazo/parto/puerperio. no tuvo una significancia tan importante en otros estudios como en el nuestro; seguido de enfermedades del sistema respiratorio y tumores $(11,8 \%)$ son las principales causas de muerte según causa básica de muerte consignada en la autopsia. Honduras presenta alta tasa de mortalidad materna (73 muertes maternas por cada $10000 \mathrm{~m}$ nacidos vivos) 20 reflejado al ser la principal causa básica de muerte dentro de la muestra ob- tenida. Este hallazgo también es influenciado por un mayor porcentaje de mujeres dentro de la muestra y una muerte materna implica una autopsia reglamentaria en Honduras. De acuerdo a la edad, la bibliografía establece que las causas externas inciden en la población más joven y los tumores en los mayores ${ }^{2}$, $3,17,19,21$.

Discrepancias Diagnósticas

El rango normal de discrepancias a nivel mundial (Clase I y Clase II de la Clasificación de Goldman) suele variar de 16\%-57\%; en Latinoamérica varía entre 6-40\%. En Costa Rica el porcentaje de discrepancia fue de $48 \%{ }^{2-10,15,16}$. La discrepancia diagnóstica detectada en nuestro estudio fue de $43,9 \%$ de discrepancias mayores (Clase I, Clase II) y 10,6\% de discrepancias menores. (Ver Tabla 4)

La concordancia fue valorada en la clase $\mathrm{V}$ (sin discrepancia diagnóstica) siendo de $54,5 \%$. El año con mayor discrepancia fue el $2014(66,7 \%)$ con un decremento significativo durante el $2015(30,8 \%)$ y el transcurso del 2016 hasta el mes de junio (18,2\%), estableciéndose correlación estadística significativa entre año y discrepancia diagnóstica $(\mathrm{p}=0,025)$. Los posibles factores asociados a errores diagnósticos son: Falta de práctica, interés en la recolección de información clínica, historia clínica incompleta o errónea, marcha diagnóstica incorrecta, exámenes complementarios y de imagen no solicitados o mal interpretados; cierre prematuro en un solo diagnóstico. A la vez, se han identificado factores externos como el poco abastecimiento de recursos, sistema institucional precario que llevan al retraso del diagnóstico ${ }^{2-5,21,22}$.

Los servicios médicos con mayor discordancia diagnóstica registrados en la literatura son Unidad de Cuidad Intensivos, Medicina Interna y Cirugía15. La sala de servicios hospitalarios con la mayor tasa de discrepancia diagnóstica en nuestro estudio, es Medicina Interna (71,2\%), que también presenta el porcentaje de defunción más alto $(67,18 \%)$, seguida en orden descendente por el servicio de Ginecoobstetricia $(19,2 \%)$ con porcentaje de defunción de $20,58 \%$. No se observó una correlación estadísticamente significativa $(p>0,05)$ entre las variables "servicios hospitalarios" con "discrepancia diagnóstica" $(\mathrm{p}=0,849)$. Esto debido a que es la sala de servicios hospitalarios con mayor referencia intrahospitalaria, sugiriendo que las discrepancias pudieron haber ocurrido en las salas de ingreso, pero esto no se especificó en los protocolos de autopsia.

Los estudios complementarios sirven de apoyo para poder llegar a un diagnóstico certero y oportuno ${ }^{11,12}$. Los protocolos de autopsia reportan la realización de estudios complementarios básico siempre, 
encontrándose discrepancia diagnóstica en 46,3\% de ellos. El uso de estudios de imagen aumentó del 2012-2016 (17,2\%) la discrepancia a pesar de su uso $y$ al prescindir de ellos es de $78,90 \%$ y $78,80 \%$ respectivamente, no hay diferencias considerables en las cifras de discrepancia y uso de estudios de imagen. El uso de estos estudios complementarios y tecnologías nunca podrán sustituir la capacidad holística que demuestra el clínico ${ }^{3,7,13}$.

Se consignó diagnóstico macroscópico en todos los protocolos, de estos $46,3 \%$ presentan discrepancia diagnóstica, su realización no genera diferencias considerables en cuanto a la misma. En los protocolos que se realizó diagnóstico microscópico se demostró que 19,3\% tenían discrepancia y se observó una correlación estadística significativa $(\mathrm{p}<0,05)$ entre las variables "diagnóstico microscópico" con "discrepancia diagnóstica” $(\mathrm{p}=0,002)$. Se observó que, en los años 2012-2014, con menos diagnósticos microscópicos consignados (34\% en total), fue en el que se observó mayor discrepancia diagnóstica (Promedio de 56\%) a diferencia del 2015-2016, con aumento de los protocolos con diagnóstico microscópico consignados $(65 \%)$ y un porcentaje mucho menor de discrepancias (Promedio de 23\%). Se confirma lo recalcado por la literatura; la consignación del diagnóstico microscópico, es significativo al momento de la presencia de discrepancia diagnóstica 8. Existe un consenso de los estudios que evaluaron los principales diagnósticos con discrepancia: Enfermedades pulmonares (Infecciosas, embolia pulmonar), infarto de miocardio, patología cerebro-vascular, peritonitis (abdomen agudo) y neoplasias. El orden de frecuen-

\section{REFERENCIAS}

1. Vítolo F. Responsabilidad Profesional del Patólogo. Biblioteca Virtual NOBLE. [Internet]. 2014. [consultado $21 \mathrm{sep}-$ tiembre 2016]. Disponible en: http://www.noble-arp.com/src/ img_up/10022015.0.pdf

2. Bürgesser $M$, Calafat $P$, Campos D, Diller A. Discrepancias entre diagnósticos clínicos y hallazgos de autopsia. Rev. Medicina Buenos Aires. [Internet]. 2011; [consultado 21 septiembre 2016]: 71(2): 135-138. Disponible en:http://medicinabuenosaires. com/revistas/vol71-11/2/v71_n2_p.135-138.pdf

3. Coradazzi A.L, Morganti A.L.C, Montenegro M.R.G. Discrepancies between clinical diagnoses and autopsy findings. Braz $\mathrm{J}$ Med Biol. [Internet]. 2003; [consultado 21 septiembre 2016] 36 (3): 385-391. Disponible en: http://www.scielo.br/scielo.php?pid=S0100879X2003000300014\&script=sci_abstract [Inglés].

4. Roulson J, Benbow E.W, Hasleton P.S. Discrepancies between clinical and autopsy diagnosis and the value of post mortem histology; a meda-analysis and review. Histopathology. [Internet]. 2005; [consultado 21 septiembre 2016]. 47(6):551-559. Disponible en: https://www.ncbi.n/m.nih.gov/pubmed/16324191 cia varía entre los distintos estudios. Estas causas se han comprobado en diversos estudios realizados en países latinoamericanos como: Colombia, México, Argentina, Brasil, Costa Rica, Cuba 2-5, 8-10, ${ }^{26}$. Nuestros resultados concuerdan con estudios internacionales con enfermedades pulmonares e infecciosas, revelando que las patologías mal diagnosticadas con frecuencia fueron: Glomerulonefritis, bronconeumonía (cada una con $5,4 \%$ ), neumonía y meningitis aguda (ambas con 3,6\%).

Las limitaciones del presente estudio, son la falta de revisión de historias clínicas de acuerdo a los protocolos de autopsia, en la etapa de recolección de datos, no se especificó en varios protocolos de autopsia: salas de ingreso del paciente, sala donde falleció o si hubo traslados entre salas. La mayoría de protocolos de autopsia no incluían la causa de solicitud de dicha autopsia. Los protocolos de autopsia presentaban escasa información en cuanto a la evolución intrahospitalaria de los pacientes dentro de cada una de las salas.

No declaramos conflictos de intereses.

\section{AGRADECIMIENTOS}

Al Departamento de Patología, por permitir el acceso a su archivo y así desarrollar la investigación. Al Dr. César Durán, por su apoyo y asesoría desde el inicio de la investigación. A la Dra. Gloria Rivera, por brindarnos la oportunidad de aportar al desarrollo de nuestro Hospital Escuela Universitario con esta investigación.

[Inglés].

5. Wittschieber D, Klauschen F, Kimmritz A, Winterfeld M, Kamphues $\mathrm{C}$, Scholman $\mathrm{H}$ et al. Who is at risk for diagnostic discrepancies? Comparison of pre and postmortal diagnoses in 1800 patients of 3 medical decades in east and west Berlin. PLoS ONE [Internet]. 2012 [consultado 17 Septiembre 2016];7(5). Disponible en: https://www.ncbi.nlm.nih.gov/pmc/articles/PMC3358345/. [Inglés].

6. Ioan B, Alexa T, Alexa I. Do we still need the autopsy? Clinical diagnosis versus autopsy diagnosis. Romanian Society of Legal Medicine [Internet]. 2012 [consultado 17 Septiembre 2016]; 20(4). Available from: $h$ ttp://www.rjlm.ro/doc/307-312dowestillneedtheautopsy.pdf. [Inglés].

7. Lobo S, Maluf Cury P, Fusco Fares A. Clinical-pathological discrepancies in critically ill patients with difficult premortem diagnoses. Bras Ter Intensiva. [Internet]. 2011 [consultado 21 Septiembre 2016]; 23(4):442-447. Disponible en:http://www.scielo. br/pdf/rbti/v23n4/en_a08v23n4.pdf [Inglés].

8. Urbina A., Rivas B., Antúnez H. Diagnóstico post mortem de 
neoplasias no sospechadas clínicamente. Rev. cienc. forenses Honduras [Internet]. 2016 [consultado 20 Septiembre 2016]; 2(1):3 12. Disponible en: $h t t p: / / w w w . b v s . h n / R C F H / p d f / 2016 / p d f /$ RCFH2-1-2016-3.pdf

9. Sblano, S., Arpaio, A., Zotti, F., Marzullo, A., Bonsignore A. Dell'Erba, A. Discrepancies between clinical and autoptic diagnoses in Italy: evaluation of 879 consecutive cases at the "Policlinico of Bari" teaching hospital in the period 1990-2009. Ann. Ist. Super. Sanità, [Internet] 2014. [consultado 21 Septiembre 2016]; 50(1): 44-48. Disponible en: http://www.scielosp.org/scielo. php?script=sci_arttext\&pid=S0021-25712014000100007. [Inglés].

10. Gonzales K, Hernandez D, Pedraza N. Compatibilidad entre el diagnóstico clínico y anatomopatológico en los servicios del Hospital Universitario Arnaldo Milián Castro. Revista Cientifica Villa Clara [Internet]. 2014 [consultado 21 Septiembre 2016];18(4):163-170. Disponiblle en: http://scielo.s/d.cu/pdf/ $m d c / v 18 n 4 / m d c 04414 . p d f$

11. Díaz J, Gallego B, León A. El diagnóstico médico: bases y procedimientos.Rev Cubana Med Gen Integr. [Internet]. 2006 [consultado 20 Septiembre 2016];22(1). Disponible en: http://bvs. sld.cu/revistas/mgi/vol22_1_06/mgi07106.htm

12. Capurro D, Roda G. El Proceso Diagnòstico. Rev. méd. Chile. [Internet]. 2007; [consultado 20 Septiembre 2016] 135(4):534-8 Disponible en: $h t t p: / / w w w . s c i e l o . c l / s c i e l o . p h p ? s c r i p t=s c i \_a r t t e x t \& p i d$ $=$ S0034-98872007000400018

13. Arce Mateos F, Fernández Fernández F, Mayorga Fernández M Gómez Román J, Val Bernal J. La Autopsia Clínica. REA: EJAutopsy. [Internet]. 2009. [consultado 18 septiembre 2016]; 7(1), 3-12. Disponible en: $h t t p: / / r e a . u n i n e t . e d u / i n d e x . p h p / e j a u t o p s y /$ article/viewArticle/34

14. Valdez-Martínez E., Array-Lonagómez E., Lándero-López L. Concordancia entre el diagnóstico clínico y el patológico por necropsias. Salud Pública Mex [Internet]. 1998; [consultado 18 septiembre 2016] 40(1): 32-37. Disponible en:http://www.scielosp. org/pdf/spm/v40n1/Y0400105.pdf

15. Casco A., Mita L. Estudio de la discrepancia entre diagnóstico de defunción clínicos y los hallazgos de autopsia en el Hospital Calderón Guardia. Acta méd. costarric [Internet] 2003 Mar consultado 21 Septiembre 2016]; 45(1): 15-19. Disponible en: $h$ ttp://www.scielo.sa.cr/scielo.php?script=sci arttext\&pid=S0001-60022003000100004\&Ing=en.

16. Durruthy O., Sifontes M., Martínez C., Olazábal A. Del certificado de defunción al protocolo de necropsias: causas básicas de muerte. AMC [Internet]. 2011 Jun [consultado 21 Septiembre 2016]; 15(3): 542-552. Disponible en: $h t t p: / /$ scielo.sld.cu/scielo.php?script=sci_arttext\&pid=S102502552011000300011\&lng=es.

17. OMS | Estado de la salud en el mundo: nuevo estudio sobre la carga mundial de morbilidad. [Internet]. OMS. Ginebra: BoudouJacobs N.; 2008 [consultado 1 Octubre 2016]. Disponible en:http:// www.who.int/mediacentre/news/notes/2008/np11/es/

18. Córtes A, Botero M, Carrascal E, Daza Y, Donado P. Concordancia entre el diagnóstico clínico y hallazgos de autopsia en dos períodos en el Hospital Universitario del Valle, Cali. Colom- bia Médica [Internet]. 2004; [consultado 22 septiembre 2016] 35(3):139-144. Disponible en: http://bibliotecadigital.univalle. edu.co/bitstream/10893/6723/1/Concordancia\%20 entre\%20el\%20diagnostico.pdf

19. Instituto Nacional de Estadística de España. Defunciones según la Causa de Muerte Año 2013 [Internet]. 2015 [consultado 18 Octubre 2016]. Disponible en: http://www.ine.es/prensa/np896.pdf

20. Honduras no cumplirá meta de mortalidad materna. El Heraldo. 28/04/2015. País:8.

21. Vítolo F. Errores Diagnósticos: Por qué se producen y pautas para evitarlos. Biblioteca Virtual NOBLE. [Internet]. 2011 [consultado 21 Septiembre 2016]. 59. Disponible en URL: http://www. nobleseguros.com/ARTICULOS_NOBLE/59.pdf

22. Schwanda-burger S., Moch H., Muntwyler J., Salomon F. Diagnos tic errors in the new millennium: A follow-up autopsy study. Modern patology. [Internet] 2012. [consultado 20 Septiembre 2016] 25: 777-783. Disponible en: http://www.nature.com/ modpathol/journal/v25/n6/pdf/modpathol2011199a.pdf [Inglés].

23. Rayego J., Rodríguez F., Mayoral L, Álvarez A, Najarro F. Mortalidad hospitalaria en el Servicio de Medicina Interna de un hospital de primer nivel. An. Med. Interna [Internet]. 2006 [consultado 1 Octubre 2016];23(9):406-410. Disponible en: http:// scielo.isciii.es/scielo.php?script=sci_arttext\&pid =S0212-71992006009900002

24. Rampatige R., Mikkelsen L., Hernandez B., Riley I., López A. Estadísticas hospitalarias sobre las causas de defunción: ¿qué hacer con ellas? Boletín OMS. [Internet]. 2014; [consultado 1 Octubre 2016] 92(1):3-3A Disponible en: http://www.who.int/bulletin/ volumes/92/1/13-134106/es/

25. Gomero P, Ríos R, Aguilera D. Causas de muerte más frecuentes en el cuerpo de guardia. Medisan [Internet]. 1999 ; [consultado 1 Octubre 2016] 3(3):1620. Disponible en: http://bvs.s/d.cu/revistas/san/ vol3_3_99/san04399.htm

26. Borrell-Carrió F, Páez Regadera C, Suñol Sala R, Orrego Villagan C, Gil Terrón N, Martí Nogués M. Errores clínicos y eventos adversos: percepción de los médicos de atención primaria. Aten Primaria.[Internet] 2006 [consultado 21 septiembre 2016];38(1):2532.Disponible en:http://www.elsevier.es/es-revistaatencion-primaria-27-articulo-errores-clinicos-eventosadversos-percepcion-los-medicos-13090027\#elsevierl temBibliografias 